\title{
A virtual tabletop workspace for the assessment of upper limb function in Traumatic Brain Injury (TBI)
}

\author{
Peter H. Wilson, Jonathan Duckworth, Nick Mumford, Ross Eldridge, Mark Guglielmetti, Patrick \\ Thomas, David Shum, and Heiko Rudolph, Member, IEEE.
}

\begin{abstract}
Traditional methods of movement assessment in clinical rehab are often labor intensive and provide a limited number of outcome variables for tracking recovery. Entry level virtual reality (VR) systems afford new possibilities for systematic assessment and treatment. This paper describes the development of a virtual tabletop environment for the assessment of upper limb function in Traumatic Brain Injury (TBI). The system is designed to present realistic virtual workspaces and to measure performance at both a functional and kinematic level. In addition, we incorporate the use of Tangible User Interfaces (TUIs) as a means of integrating performance with the workspace. Unlike top-end movement analysis systems, the experimental system utilizes readily available computing technologies: mid-range PC, LCD panels, stereo camera, Virtools software, and TUI enabled by Wii Remote, Wii Sensor Bar $\left(\right.$ Nintendo $^{\mathrm{TM}}$ ) and passive markers. The combination of visionbased marker tracking with a low cost game controller (viz Wii system) provides a stable and accurate means of tracking the TUI on the virtual workspace, and for interactivity within this space. The system provides a compelling sense of realism for the performer and an innovative means of assessing movement capabilities over time.
\end{abstract}

\section{INTRODUCTION}

$\mathrm{T}$ RADITIONAL approaches to movement assessment in clinical settings can be labor intensive and show questionable reliability [1]. While the use of top-end movement analysis systems can track complex movements and streamline data collection, the cost of many of these systems is prohibitive. Moreover, these systems are not designed to directly control task parameters or any stimulus display. Alternatively, the application of virtual reality to assessment and rehabilitation has gained considerable impetus in recent years, and presents a range of possibilities for flexible, low

Manuscript received April 14, 2007. This work was supported in part by an Australian Research Council (ARC) Linkage Grant LP0562622, and Synapse Grant awarded by the Australia Council for the Arts.

P.H. Wilson is with the Division of Psychology, RMIT University, Melbourne, Australia. (Phone 613-9925-2906; fax: 613-9925-3587; email: peter.h.wilson@rmit.edu.au).

J. Duckworth is an artist and $\mathrm{PhD}$ candidate with the School of Creative Media, RMIT University, Melbourne Australia.

N. Mumford is a PhD candidate with the Division of Psychology, RMIT University

R. Eldridge and H. Rudolph are with the School of Electrical and Computer Engineering, RMIT University, Melbourne Australia

M. Gugliemetti is an artist and Lecturer with the School of Art and Design, Monash University.

P. Thomas is with the School of Curriculum, Teaching and Learning, Griffith University, Brisbane, Australia (email: p.thomas@griffith.edu.au).

D. Shum is with the School of Psychology, Griffith University, Brisbane, Australia (email: d.shum $@$ griffith.edu.au). cost solutions, as well as more sophisticated immersive systems [2]. In this paper we describe the development of one such system (called Elements) for use with Traumatic Brain Injury (TBI); Elements uses low-end technologies to achieve a suitable level of precision and adaptability.

Experimental work reveals that TBI patients frequently show impaired motor planning due to diffuse damage of brain networks responsible for forming motor intentions and their translation into movement (i.e., reciprocal loops involving premotor cortex, parietal cortex, basal ganglia, and cerebellum) [3]. This is manifest by abnormal kinematics: increased response time especially when compensating for target shifts, poor trajectory control, greater response variability, and so on [4]. However, the particular constellation of deficits at a kinematic and functional level may vary from one person to another, hence, the importance of assessing individual differences and within-person change over time. As well, research has demonstrated that movement skill in this population can be re-trained by shaping motor planning functions [5]. It is thus important to monitor these changes in response to therapy. VR-based technologies provide an excellent medium for creating environments within which planning processes and functional skill can be assessed, as well as for designing a graded set of rehabilitation activities; virtual environments (VEs) can be used to engage the TBI patient, enhance motivation, automate data collection, and provide greater control over the presentation of task constraints [6].

The conceptual development of the Elements system is consistent with emerging trends in Human Computer Interaction $(\mathrm{HCI})$ and the neuroscience of movement $[7,8]$. Our conceptual approach has blended (ecological) motor learning theory with interaction design principles to inform the creation of flexible and engaging workspaces for clinical assessment and rehab. Previously we presented a broad conceptual model to show that movement behaviour is given by the interaction of task, person, and individual constraints [7], and can be assessed at different levels (from neurocognitive primitives to functional outcomes). More specifically, the concept of affordance proposed by Gibson [9] and other ecological theorists (e.g., Turvey) has informed the way we conceive of the relationship between performer and workspace $[10,11]$. Affordance refers to the opportunities for interaction that meaningful objects provide in our immediate environment and in relation to our sensorimotor capacities; the perceptual properties of different objects and events are, thus, 
mapped fairly directly to the action systems of the performer $[9,12]$. The affordances offered by the VE and tangible user interfaces (TUIs) are designed to engage the patient's attention to the movement context and the immediate possibilities for action. So, rather than embedding virtual objects in a virtual world, we use real objects and a direct mode of interaction. In short, the ecological approach has a lot to commend itself by not drawing an artificial distinction between the performer and the natural constraints of his performance. Advances in interactive design also accord with this interactive or embodied view of performance.

With the emergence of mobile computing and TUIs, digital environments are able to transcend the "flat world" of the computer display screen and become part of the user's physical space. Case studies in the interactive design of artists $[13,14]$ and of theorists involved with HCI [15-17] support this trend. They argue that the basis of interaction design should be a first-person, lived experience and its relation to the environment. These case studies indicate new possibilities for movement based interactivity [15-17]. For example, technologist and artist Myron Krueger pioneered novel forms of HCI using video capture techniques that interpret the body's position relative to a computer simulated graphical environment called VIDEOPLACE [13]. Our group has explored how to best use the latest movement sensing technology to track real objects that, in turn, mediate the form of interaction between performer and environment. For the purpose of movement assessment, these virtual, interactive spaces can be presented in standardized formats, with task parameters varied systematically. As a rehab space, the VEs can be adapted to patients' individual needs, and varied according to their rate of progress in therapy. For instance, these technologies can enable motor learning, re-learning and expression by providing a mode of interaction that reinforces the patient's sense of position in space via augmented feedback $[6,18]$.

A crucial part of an affordable computer-based movement assessment system is the type of interface that mediates the interaction between the performer (or patient) and the virtual environment. The standard gaming interface that most people are familiar with is the joystick. This is designed to manipulate an avatar in a virtual space and, thus, can still be regarded as an indirect form of communication. By comparison, TUIs provide a more direct form of interaction. Here a real object is manipulated which then maps directly onto meaningful changes in the structure of the virtual environment $[15,17]$. For example, the movement of a real chess piece might be tracked using a marker system and camera which then mediates interaction with virtual pieces displayed on a simulation. Or, in our case, a simple object (hereafter labeled simply as Object) functions as a TUI; it is manipulated in response to location cues in the $\mathrm{VE}$ while being position tracked itself.

Factors that should be considered when choosing an object tracking method include: cost, functionality, sampling rate, resolution, and reliability. Movement analysis systems using visual tracking are deemed too expensive for most clinical settings; and, the less expensive electromagnetic systems are encumbered by wired sensors (e.g., Polhemus Patriot) [19]. For small workspaces, however, other vision-based solutions are now viable. Our group has incorporated the use of a high resolution stereo camera to provide seamless tracking of a TUI Object equipped with passive markers. In addition, we have integrated new game technologies that make use of infra-red tracking; that is, we have used Nintendo Wii Remotes, the primary controller used for the Nintendo Wii games console [20]. The system affords a low cost and yet potentially quite flexible alternative. One important issue for VR-based assessment is the relative performance of these emerging systems. This paper describes how these technologies have been used to development a powerful VR-based movement assessment system (Elements) that incorporates a virtual tabletop environment and TUIs.

\section{EXPERIMENTAL SYSTEM HARDWARE}

\section{A. Overview}

The hardware configurations of most rehabilitation systems generally fall into two categories: systems with accurate human motion tracking that encumber the user (e.g., wired gloves or specialized motion tracking suits), or unencumbered systems that offer more generalized gesture-based interaction. The Elements system was designed to achieve a balance between these two constraints. Our aim was to create a rehabilitation system that provides practical, usable real-time tracking without encumbering the patient. To achieve this end we use a constrained interaction area (the LCD panel), a stereo camera, and TUI (enabled by the Wii Remote game controller) for reach-to-grasp, lift, and placement tasks.

\section{B. PC Hardware}

The Elements system runs on off-the-shelf PC hardware. The development system runs on a Shuttle PC that is more portable than a standard ATX case thanks to its small size (Dimensions: $21.5 \times 21.5 \times 32 \mathrm{~cm}$ ). The $\mathrm{PC}$ has an AMD Athlon64 X2 Dual Core $4400+(2.21 \mathrm{GHz})$ processor and $2 \mathrm{~GB}$ RAM. The PC is equipped with an nVidia GeForce 7800 graphics card which features two dual link DVI ports for monitor connection. In order to connect a third screen for the clinician's interface, a video image splitter is used (Matrox DualHead2Go Digital Edition) [21]. The output from a single DVI port is then split into separate image streams for both vertical and horizontal LCD panels.

The small size of the Shuttle PC can be easily concealed from the user's view. In this way the only possibilities for interaction that is visible to the user is the screen itself and the TUIs, which reduces any distraction. Other similar systems utilize rear projection displays rather than LCD panels. We chose to investigate using LCD panels to improve the portability of the system and reduce set-up time.

The VE is displayed onto a 40" LCD panel placed horizontally on a tabletop surface, with audio cues via stereo speakers. The patient interacts with the system by moving 
simple objects across the LCD display; the Wii Remotes are embedded inside the objects. These objects are tracked by a stereo camera and infrared light system mounted $700 \mathrm{~mm}$ above the centre of the display. A vertical 40" LCD panel behind the horizontal screen can display performance information (i.e., knowledge of results - KR) for the performer when not in interactive mode and graphical summaries of their progress over time. A separate 17" monitor, wireless keyboard and mouse are connected to the system to facilitate clinician control.

\section{Interface Design and Object Tracking}

The Elements system combines a vision-based tracking solution and wireless game controllers in order to achieve realtime interaction without encumbering devices or wires. It uses a camera system from PointGrey ${ }^{\mathrm{TM}}$ called Bumblebee 2 which has pre-calibrated stereo cameras for accurate depth measurement [22]. Any tracked movement can be easily correlated to real spatial units. This system comes preconfigured, and is highly flexible. An IEEE1394 connection allows for full color $640 \times 480$ pixel images from both cameras at $48 \mathrm{~Hz}$

In addition, the Elements main physical interface uses Nintendo's hand held Wii Remotes adapted for Microsoft Windows XP using joystick software emulation (PPJoy and GlovePIE)[23] and a Bluetooth wireless link to communicate with the PC. The main advantages of using the Wii Remote are the availability, low cost, adaptability, and ease of calibration. The Wii Remotes are embedded within Objects that are cold cast and encased in a tailored silicon rubber; this outer casing protects the Wii Remote and LCD screen from accidental damage whilst also creating a low-slip tactile grip surface. The texture is akin to rubber, now often found coated on plastic drink bottles.

The main features of the Wii Remote are its motion sensing capabilities that enable the clinician to measure the end-point motion of the hand held controller and, thus, the patient's ability to manipulate the Object. The Wii Remote can sense both rotational orientation and translational acceleration along $\mathrm{x}, \mathrm{y}$, and $\mathrm{z}$ axes via a three-axis linear accelerometer embedded in the controller. The device is rated to measure accelerations over a range of at least $+/-3 \mathrm{~g}$ [24].

The Wii Remote also features a 1 mega pixel monochrome sensor with an Infrared (IR) filter mounted in front; the filter is positioned at the top end of the device. The IR sensor is used to track two IR LED points at each end of a Sensor Bar provided with the Wii console. The Sensor Bar is mounted above the horizontal LCD screen orientated downward and fixed to the Bumble Bee stereo camera. The fixed spacing between LEDs on the bar enable the Wii Remote to calculate its distance relative to the IR light points. By tracking the locations of these two IR light points in the sensors 2D field of view, the system can derive more accurate positional information. The tilt and rotation of the Wii Remote with respect to the ground can also be calculated from the relative angle of the Sensor Bar LED lights and are combined with the data sensed by the accelerometer to provide six degrees of freedom in a three dimensional workspace. The entire hardware configuration is presented schematically in Figure 1.

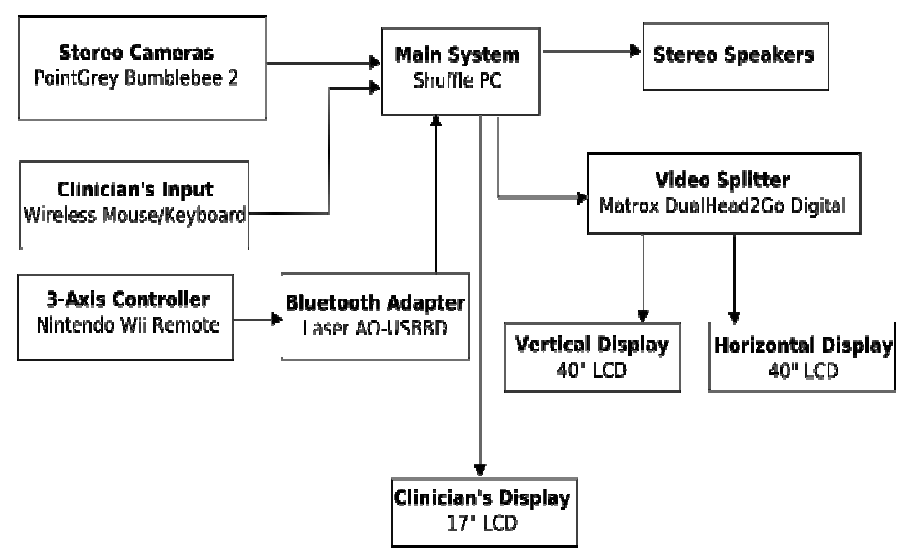

Fig. 1: Hardware Functional Block Diagram.

\section{EXPERIMENTAL SYSTEM SOFTWARE}

The patient interacts with the system by moving the Object placed on the horizontal LCD surface. They are cued from within the VE.

Markers placed on top of the Object are used as a point of reference for the vision-based tracking camera mounted above the screen. This permits tracking of object position and orientation without requiring active (or powered) markers. Reliability testing with a single camera ARToolkit system showed this set-up to be too inaccurate and error-prone for the hardware configuration. We then trialed the Compass $3 \mathrm{D}$ software from PointGrey ${ }^{\mathrm{TM}}$. This uses stereo camera information to provide more accurate and stable tracking of fiducial markers. This technique of tracking a TUI provides effective end-point data of patient's arm motion during the training scenarios. Our system is not designed to provide multi-joint data, unlike conventional motion analysis; this limits our ability to monitor compensatory movements.

We have also completed some preliminary work on a marker-less tracking system for hand/arm movements (without objects). Testing of PointGrey's Triclops stereo vision library has produced full image stereo disparity at reasonable resolution and frame rate. However, this means of tracking is a long term venture and beyond the scope of the present paper. Our main priority has been to achieve stable object tracking.

The virtual environments have been developed using Virtools Dev 4.0 (TM) [25]. Virtools provides a suite of software tools to develop and deploy real-time interactive applications for the web, CD ROM and Virtual Reality multiscreen applications. The graphical user interface is intuitive for both programmers and non-programmers alike, making the software an ideal choice within our multidisciplinary team of researchers.

Virtools building blocks have been constructed to provide position and orientation data from the Compass3D based tracking system and Wii Remotes. The system is calibrated by 
placing the TUI within the centre of the screen. The software reads this initial position using the Compass $3 \mathrm{D}$ system and estimates the size and location of the horizontal display. The position of the Object can then be read from the Compass3D system every camera frame (approx. $50 \mathrm{~Hz}$ ) to provide realtime data. The precision of positional data is currently rated at $<+/-3 \mathrm{~mm}$. The Object acts as the main interface to the Elements system, much in the same way as a mouse and keyboard. Movements are updated every frame and a 'click' is triggered whenever the user moves the object below a certain threshold in the $\mathrm{z}$ (vertical) plane (i.e., placement on the screen).

\section{IMPLEMENTATION}

\section{A. Workstation design}

The Elements workstation (Figure 2) is arranged on a desk $1250 \mathrm{~mm}$ wide, $900 \mathrm{~mm}$ deep, and $650 \mathrm{~mm}$ high. On the desk are positioned two 40" LCD monitors, one mounted horizontally and the other vertically. The therapist has a separate desk to the side of the workstation, and controls the program from this location. The therapist can pause the program from their control station at any time to administer individual instructions depending on the participant's level of proficiency and stage of recovery. Once the program is paused

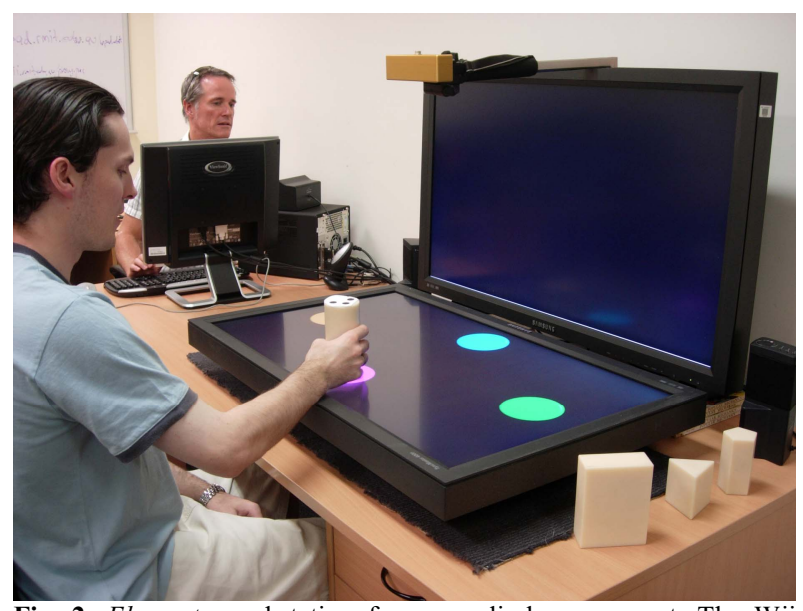

Fig. 2: Elements workstation for upper limb assessment. The Wii Sensor bar is mounted directly behind the stereo camera, from the user's perspective.

the sequence can be resumed, restarted, or aborted.

\section{B. Assessment Tasks - Rationale and implementation}

The ability to reach, grasp, and manipulate objects requires not only a base physical capability, but an ability to plan movements under varying task and environmental constraints. The performer needs to map object properties and spatial location onto intrinsic motor coordinates in order to achieve a movement goal [3]. A complex set of control processes govern this mapping and aberrations of movement kinematics will reflect deficits in planning. Our system is designed to assess both (basic) kinematics and functional performance, and to vary task constraints in a graded way in order to challenge these planning functions [26].
The Elements program consists of two main assessment tasks, each with two sub-tasks. Each requires that participants move a cylindrical Object (63mm diameter, $130 \mathrm{~mm}$ high) to locations in the VE. Initially the Object is placed on the home base. Subsequently, a target, seen on the horizontal display, is cued by a flashing border around the icon, and the object is moved to that location. Participants return their hand to the home base, which will then be highlighted, and the object returned. And, the next target in the series is cued. To complete these activities participants must plan movements in peri-personal space, both to and from a body-centred axis. Speed and accuracy of movement are synonymous with movement skill [3], and patients are assessed accordingly. They are instructed to complete the movements as quickly as possible, and position the Object in the centre of the target. At the completion of an assessment session, performance data can be presented in graphical form on the vertical LCD: completion time, path length, and number of corrective movements, and placement accuracy. The rehab protocol (to be presented elsewhere) makes judicious use of this feedback; knowledge of performance and results is pivotal to the refinement of general motor planning and skill, and the modification of internal models for action in particular [26]. The assessment tasks in the Elements program, described below, are designed hierarchically to promote flexibility and complexity of motor planning.

Task 1. Task 1(a) consists of the home base and three potential movement targets, $78 \mathrm{~mm}$ in diameter (see Figure 3). These target locations were selected to be a comfortable reaching distance from the home base. The targets are highlighted in a fixed order (west, north, east). As the sequence is predictable and of fixed distance, minimal demands are placed on movement planning functions. Task 1(b) has the same target configuration, but they are highlighted in random order. Thus, spatial uncertainty is introduced, placing a marginally higher demand on attention and response flexibility. The patient is asked to complete a set of nine movements (three per target), the exact number of sets dependent on their stage of recovery.

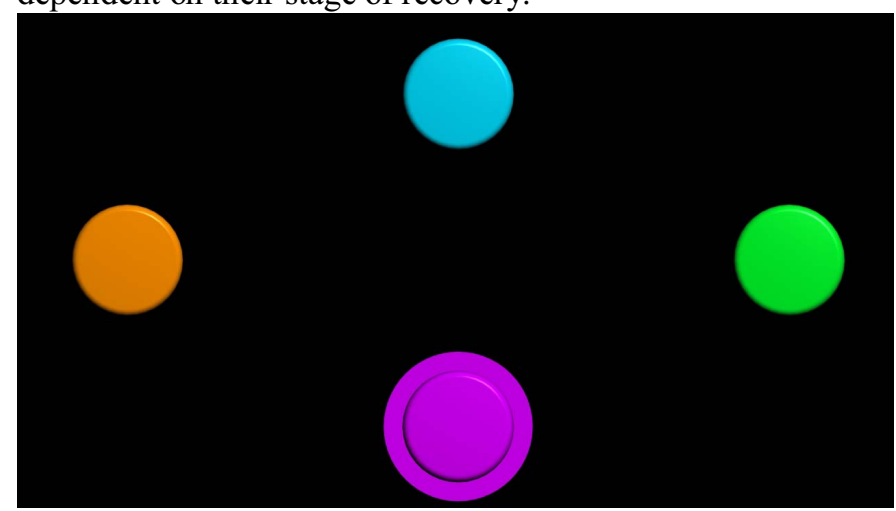

Fig. 3. Positioning of the north, east, west, and home base targets for Task 1(a) and $1(\mathrm{~b})$.

Task 2. Task 2(a) begins with a blank screen. A target circle then appears randomly in each of nine locations. These locations are configured along three radials emanating from 
the home base; i.e., original locations (Figure 3) plus an additional two for each radial path. This configuration enables target trajectory and distance to be varied over trials. Thus, participants are required to perform a movement "somewhere" in the VE, necessitating more flexible control of trajectory and end-point. The ability to alter movements flexibly in response to changing environmental constraints is a central aspect of motor planning and internal modeling [26]. This task set-up enables these control functions to be assessed. The simple but dynamic layout of the virtual workspace is designed to draw the user's attention unambiguously to task goals. The shifting location of targets in an uncluttered space provides a compelling sense of engagement for the user. We believe that simple tasks of this type are fully transferable to the real world.

Task 2(b) uses the same target positions as 2(a); however, additional distractor targets appear - a hexagon, triangle, and rectangle. All "targets" are presented in blue. Participants are instructed to place the cylindrical Object on the circle target only. If placed incorrectly the distractor turns red, and the Object must be moved back to the home base. Thus, Task 2(b) places a greater load on response selection and movement inhibition. That is, patients are required to attend closely to task-relevant cues, inhibiting a response to what may be considered a compelling signal in many instances.

Measured Variables. The speed, precision, distance moved, and accuracy of target selection data recorded by the Elements program will allow motor performance to be monitored longitudinally. Improved motor function will be demonstrated by reduced movement speed, improved accuracy, reduced distance the object is moved, and increased accuracy of target selection. Forthcoming psychometric analysis will validate the Elements data in comparison to standardized measures. Furthermore, comparison of data across activities will provide performance data as a function of task constraint.

\section{DISCUSSION}

A blending of ecological approaches to movement and new thinking in HCI can greatly inform our approach to movement assessment and rehabilitation. By understanding the nature of embodied interaction and the greater affordances for human movement and expression enabled by the latest movement sensing technology, new design possibilities for movement based interaction can be explored, particularly in relation to TBI assessment and therapy.

We have developed novel ways of using VR-based technologies to build a flexible, automated, and relatively lowcost system of assessment. The Elements system takes the concept and application of TUIs to another level, supporting clinical practices; the mode of interaction that is enabled by the TUI Object is direct and compelling with respect to the virtual environment. We make novel use of game controllers and marker tracking to achieve these outcomes. The Object serves as the medium for interaction and provides accurate movement data for end-point control and for functional accuracy. This data can be collated as a time series for tracking recovery.

We envisage that our VR-based system can be easily adapted to find a broader application in the assessment of movement in other neuropsychological disorders (e.g., stroke and ischemic disorders), and movement disorders associated with aging (e.g., Parkinson's Disease and Huntington's chorea).

\section{ACKNOWLEDGMENTS}

This project was funded by an ARC Linkage Grant (LP0562622) and Synapse Grant from the Australia Council for the Arts.

\section{REFERENCES}

[1] B. Bobath, Adult hemiplegia: Evaluation and treatment. London: Heinemann Medical books, 1989

[2] S. Blanton, L. Porter, D. Smith, and S. L. Wolf, "Strategies to enhance mobility in traumatic brain injured patients," in Rehabilitation of the adult and child with TBI, vol. 3rd, M. Rosenthal, Ed. Philadelphia, PA, 1999, pp. 219-241.

[3] P. H. McCrea, J. J. Eng, and A. J. Hodgson, "Biomechanics of reaching: clinical implications for individuals with acquired brain injury," Disability and Rehabilitation, vol. 24, pp. 534 - 541, 2002

[4] B. A. Wilson, "Traumatic brain injury," in Comprehensive Clinical Psychology, A. S. Bellack and M. Hersen, Eds. Oxford: Elsevier Science Ltd, 1998, pp. 463-486.

[5] S. Page, P. Levine, and A. C. Leonard, "Effects of mental practice on affected limb use and function in chronic stroke," Archives of Physical Medicine and Rehabilitation, vol. 86, pp. 399-402, 2005

[6] A. A. Rizzo, M. T. Schultheis, K. A. Kerns, and C. Mateer, "Analysis of assets for virtual reality applications in neuropsychology," Neuropsychological Rehabilitation, vol. 14, pp. 207-239, 2004.

[7] P. H. Wilson, P. Thomas, D. Shum, J. Duckworth, M. Gugliemetti, H. Rudolph, N. Mumford, and R. Eldridge, "A multilevel model for movement rehabilitation in Traumatic Brain Injury (TBI) using Virtual Environments.," presented at 5th International Workshop on Virtual Rehabilitation, New York, 2006.

[8] M. Jeannerod, Motor cognition: What actions tell to the self. Oxford, UK: Oxford University Press, 2006.

[9] J. J. Gibson, The senses considered as perceptual systems. Boston: Houghton Mifflin, 1966.

[10] W. H. Warren, "Self-motivation: Visual perception and visual content," in Handbook of perception and cognition, W. Epstein and S. Rogers, Eds. New York: Academic Press, 1995.

[11] M. T. Turvey, "Preliminaries to a theory of action," in Percieving, Acting and Knowing: Toward and Ecological Psychology, R. Shaw and J. Bransford, Eds. Hillsdale, NJ: Erlbaum, 1980

[12] F. Garbarini and M. Adenzato, "At the root of embodied cognition: Cognitive science meets neurophysiology," Brain and Cognition, vol. 56, pp. 100-106, 2004.

[13] M. Krueger, Artificial Reality II. Reading, MA: Addison-Wesley Publishing Company, 1991.

[14] S. Cornock and E. Edmonds, The Creative process where the artist is amplified or superseded by the computer, vol. 6: Pergamon Press, 1973.

[15] H. Ishii and B. Ullmer, "Tangible Bits: Towards seamless interfaces between people, bits and atoms," presented at Proceedings on Human Factors in Computing Systems, 1997.

[16] P. Dourish, Where the action is: The foundations of embodied interaction. Cambridge, MA: MIT Press, 2001

[17] W. Buxton, G. Fitzmaurice, and H. Ishii, "Bricks: Laying the foundations for graspable user interfaces," presented at ACM Conference on Human Factors in Computing Systems, 1995.

[18] M. Holden, "Virtual environments for motor rehabilitation: Review.," Cyberpsychology \& Behavior, vol. 8, pp. 187-211, 2005.

[19] Polhemus Patriot, http://polhemus.com/.

[20] Nintendo Wii, http://wii.nintendo.com/controller.jsp. 
[21] Matrox DualHead2Go Digital $\quad$ Edition, http://www.matrox.com/graphics/en/gxm/products/dh2go/digital/home.p hp.

[22] PointGrey Bumblebee2, http://www.ptgrey.com/products/bumblebee2

[23] C. Kenner, GlovePIE, http://carl.kenner.googlepages.com/glovepie.
[24] Analog

Devices, http://www.analog.com/en/prod/0\%2C2877\%2CADXL330\%2C00.html

[25] Vitrools, http://www.virtools.com.

[26] D. Wolpert, "Computational approaches to motor control," Trends in Cognitive Sciences, vol. 1, pp. 209-216, 1997. 